\title{
Acinetobacter baumannii bloodstream infections in a tertiary hospital: Antimicrobial resistance surveillance
}

\author{
Najwa Al-Mously ${ }^{1}$, Ahmed Hakawi ${ }^{1,2}$ \\ 1. Faculty of Medicine at King Fahad Medical City, King Saud bin Abdulaziz \\ University for Health Sciences, Riyadh, Saudi Arabia \\ 2. King Fahad Medical City Hospitals, Riyadh, Saudi Arabia.
}

\begin{abstract}
Nosocomial bacteremia caused by Acinetobacter baumannii is of increasing concern in critically ill patients. There is a world-wide increase in the emergence of multidrug-resistant (MDR) $A$. baumannii to antimicrobials. Therefore we conducted this study to evaluate the resistance pattern of $A$. baumannii in patients with bacteremia. This is a retrospective study conducted in a tertiary hospital in Saudi Arabia. During the period from April 2006 till April 2010, data for A. baumannii isolated from blood were collected. Multidrug resistant A. baumannii was defined as resistance to three or more classes of antibiotics.
\end{abstract}

We found 191 isolates of $A$. baumannii from blood. The majority of cases were reported in surgical ICU (40.8\%). The highest resistance rates to antimicrobials were reported for cefepime (73.8\%) and piperacillin / tazobactam $(72.3 \%)$, followed by ciprofloxacin (68\%), and gentamicin (66\%). Resistance rates were also high to imipenem $(61.3 \%)$ and meropenem $(60.7 \%)$. Low rates of resistance to colistin $(0.5 \%)$ and to tigecycline (3.9\%) were observed. MDR was observed in $132(69 \%)$ of the $A$. baumannii isolates and $104(78.8 \%)$ of these MDR strains showed sensitivity only to colistin and tigecycline.

Bacteremia due to $A$. baumannii was the highest in surgical ICU. The emergence of MDR A. baumannii is increasing and the resistance rates to carbapenems are alarming. Urgent measures are in need to slow the development of MDR AB. We recommend encouraging clinicians for strict adherence to infection control

\footnotetext{
Corresponding Author

Najwa Al-Mously

Faculty of Medicine at King Fahad Medical City

King Saud bin Abdulaziz University for Health Sciences,

Riyadh, Saudi Arabia

Email: nalmously@kfmc.med.sa
} 
policies, enhancement of ongoing antimicrobial stewardship program and restrictive use of recommended antibiotics according to susceptibility testing and local guidelines. In-house surveillance is needed for the detection of resistance rates particularly MDR A. baumannii.

\section{Key words}

Acinetobacter baumannii; Cross infection and drug therapy; Bacterial infections and drug therapy; Drug resistance, multiple; Carbapenems and therapeutic use

\section{Introduction}

Members of Genus Acinetobacter are heterogenous group of non-fermentative, Gram-negative coccobacilli which have been grown from different human sources and are widespread in the environment. ${ }^{1}$ The most important species from this genus is Acinetobacter baumannii which has emerged in recent years as a major cause of healthcare-associated infection (HAI) and are responsible for serious nosocomial infections especially in intensive care units., ${ }^{2,3}$ This bacteria present a challenge to Infection Control Department at hospitals since it can be found anywhere in the hospital environment with ability to survive for prolonged periods thus potentiating its ability for nosocomial spread. ${ }^{4}$ Similarly, A. baumannii infections present a challenge to clinicians since the organism can rapidly acquire antimicrobial resistance. ${ }^{3}$ Clinical infections include pneumonia, bacteremia, meningitis, surgical site infections, skin infections, and catheter-related and urinary tract infections. ${ }^{5}$ The impact of healthcareassociated bloodstream infection on the outcome of critically ill patients has been extensively studied with documented mortality rate ranging from $19 \%$ to $35 \% .{ }^{6,7}$ A. baumannii has been reported to be a leading cause of nosocomial bloodstream infections in the intensive care unit (ICU) ${ }^{6,7}$ Risk factors correlated with bloodstream infections in ICU include patients with: immunosuppression, unscheduled admission, respiratory failure at admission, and previous antimicrobial therapy. Previous sepsis in ICU and the number of invasive procedures were also related to the development of $A$. baumannii bacteremia in this unit. ${ }^{8}$

A. baumannii can rapidly acquire antimicrobialresistance genes and thus become multidrug resistant. ${ }^{9,10}$ The carbapenems (imipenem, meropenem, and doripenem) are among the drugs of choice for treatment of these multidrug-resistant $A$. baumannii infections. ${ }^{11}$ However, carbapenem resistance is increasingly reported and has become a significant public health concern. ${ }^{12-14}$ Among the risk factors for infection with resistant $A$. baumannii is the widespread usage of these broad-spectrum antibiotics that has accelerated the evolution and spread of multidrugresistant (MDR) bacteria. ${ }^{9}$ The worldwide increase in the incidence of MDR isolates is associated with increased mortality. ${ }^{3,9}$ Most reports supported the fact that antibiotic resistance gene is the major molecular basis for drug resistance and the genetic mobile elements in the rapid acquisition of multiple antibioticresistance genes, like transposon and integron. ${ }^{14}$

In Saudi Arabia, there have been only few studies published on bacteremia caused by A. baumannii. These reports have shown the incidence and some risk factors for infection as well as antimicrobial susceptibility of this bacterium. ${ }^{15,16} \mathrm{Al}$-Tawfiq and Mohandhas reported the changing pattern of sensitivity to antimicrobial drugs in terms of the increase of resistance over the years. ${ }^{16}$ The changes in the incidence and aetiology of bacteraemia in hospitals are well documented. Sentinel surveys of bacteraemia in major hospitals should be conducted in order to detect the changing patterns of bacteraemia and antibiotic sensitivity of pathogens. We conducted this retrospective study to evaluate the rate of resistance of $A$. baumannii causing bacteraemia to the currently used antimicrobial drugs to treat this infection. In addition, to evaluate the prevalence of multidrug-resistant isolates recovered from blood sample in a tertiary care hospital in Riyadh, Saudi Arabia.

\section{Materials and Methods}

All blood culture positive for $A$. baumannii and their in vitro sensitivity to antibiotics were included for the period from April 2006 to April 2010. For each 
patient the result of one blood culture was considered. Bacteria were identified to the species level, and antimicrobial susceptibility testing was done with an automated microbiology system (Phoenix; BD). The following antimicrobial agents were tested by the minimum inhibitory concentration method with the Phoenix system: cefepime, piperacillin/tazobactam, ciprofloxacin, gentamicin, imipenem, meropenem, colistin. Quality control was performed by testing these same antimicrobials against reference strains of bacteria. The antimicrobial susceptibility of tigecycline was tested by Kirby-Bauer disk diffusion method (Oxoid, UK). When the result of the susceptibility testing comes as intermediate or resistant for Colistin or Tigecycline, an E-test was done for conformation ( $A B$ BIODISK, Sweden). The results of the antimicrobial susceptibility tests were interpreted according to the guidelines of the Clinical and Laboratory Standards Institute (formerly the National Committee for Clinical Laboratory Standards). ${ }^{17}$ In this study, multidrug resistance in $A$. baumannii was defined as resistance to 3 or more of the following antibiotics: antipseudomonal penicillins, antipseudomonal cephalosporins, antipseudomonal fluoroquinolones, aminoglycosides, and carbapenems. . $^{18,19}$

\section{Results}

From April 2006 to April 2010, a total of 191 isolate of A. baumannii were recovered from blood cultures. The total number of patients in this study was 191 patients, of which $160(83.8 \%)$ were adult and $31(16.2 \%)$ of paediatric age group, and $83(43.5 \%)$ were females and 108 (56.5\%) were males. The prevalence of $A$. baumannii in blood was mostly reported in patients in critical care units and the highest occurrences were reported with patients in main surgical intensive care unit (ICU) (40.8\%). This was followed by Oncology unit $(9.9 \%)$, Cardiac care unit (CCU) (8.4\%), Paediatric intensive care unit (PICU) (7.3\%), Neonatal intensive care unit (NICU) (6.9\%), Neurology unit (4.7\%), Main Emergency unit $(2.6 \%)$, and $19.4 \%$ were recovered from different units in the hospital. Of the total 191 A. baumannii isolates, 127 (66.5\%) were recovered from blood as pure culture. The frequency and type of the other bacteria recovered from mixed cultures are shown in Table I.
Table I. Co-infecting bacteria in 64 patients

\begin{tabular}{lr} 
Type of bacteria & No. of isolates \\
\hline Chryseomonas luteola & 1 \\
\hline Enterbacter cloacae & 2 \\
\hline Enterobacter aerogenes & 2 \\
\hline Enterococcus faecalis & 14 \\
\hline Enterococcus faecium & 5 \\
\hline Escherichia coli & 7 \\
\hline Klebsiella pneumoniae & 13 \\
\hline Morganella morganii & 2 \\
\hline Pseudomonas aeruginosa & 12 \\
\hline Stenotrophomonas maltophilia & 6 \\
\hline Total & $\mathbf{6 4}$ \\
\hline
\end{tabular}

A. baumannii showed high resistance rates to most of the tested antimicrobial drugs as shown in Figure 1. The total highest reported rates of resistance were for cefepime (73.8\%) and piperacillin/ tazobactam $(72.3 \%)$, followed by ciprofloxacin $(68 \%)$, and gentamicin $(66 \%)$. The rates of resistance to carbapenems were also high reaching $(61.3 \%)$ for imipenem and $(60.7 \%)$ for meropenem. The rates of resistance to colistin $(0.5 \%)$ and to tigecycline $(3.9 \%)$ were much lower in comparison to carbapenems. The full range of the annual antimicrobial susceptibility rate of $A$. baumannii to the tested antimicrobial drugs is shown in Table II. The trend of the annual pattern of resistance to antimicrobial drug is shown in Figure 2.

MDR A. baumannii was reported in 132 (69\%) of the total number of isolates (191). Table III demonstrates the distribution of MDR and non-MDR isolates by year. From these 132 MDR isolates, 104 (78.8\%) showed sensitivity only to colistin and tigecycline, 11 $(8.3 \%)$ showed sensitivity only to colistin, 11 (8.3\%) showed sensitivity only to carbapenems, colistin and tigecycline. There was one $(0.75 \%)$ isolate which was resistant to all tested antimicrobials and only sensitive to tigecycline (data not shown).

\section{Discussion}

A. baumannii has emerged as one of the most troublesome pathogens for health care institutions globally, targeting the critically ill hospitalized patients. ${ }^{9}$ In this study, we retrospectively reviewed 
Table II. Annual antimicrobial susceptibility rate of $A$. baumannii recovered from patients from April 2006 to April 2010

\begin{tabular}{|c|c|c|c|c|c|}
\hline Antibiotic & $\begin{array}{r}2006 \\
(\mathrm{n}=14) \\
\mathrm{N}(\%) \\
\end{array}$ & $\begin{array}{r}2007 \\
(\mathrm{n}=50) \\
\mathrm{N}(\%)\end{array}$ & $\begin{array}{r}2008 \\
(n=59) \\
N(\%)\end{array}$ & $\begin{array}{r}2009 \\
(\mathrm{n}=52) \\
\mathrm{N}(\%)\end{array}$ & $\begin{array}{r}2010 \\
(n=16) \\
N(\%)\end{array}$ \\
\hline Cefepime & $\begin{array}{r}\text { R } 8(57.2) \\
\text { I } 1(7.1) \\
\text { S } 5(35.7) \\
\end{array}$ & $\begin{array}{c}\text { R } 36 \text { (72) } \\
\text { I } 1 \text { (2) } \\
\text { S } 13 \text { (26) }\end{array}$ & 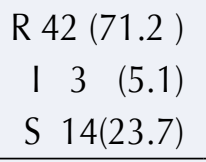 & $\begin{array}{r}\text { R } 44 \text { (84.6) } \\
\text { I } 4(7.7) \\
\text { S } 4(7.7) \\
\end{array}$ & $\begin{array}{ll}\text { R } 11 & (68.8) \\
\text { S } 5 & (31.2)\end{array}$ \\
\hline $\begin{array}{l}\text { Piperacillin / } \\
\text { Tazobactam }\end{array}$ & $\begin{array}{r}\text { R } 7(50) \\
\text { I } 1(7.1) \\
\text { S } 6(42.9) \\
\end{array}$ & $\begin{array}{l}\text { R } 36(72) \\
\text { S } 14(28)\end{array}$ & $\begin{array}{l}\text { R } 40(67.8) \\
\text { I } 3(5.1) \\
\text { S } 16(27.1) \\
\end{array}$ & $\begin{array}{r}\text { R } 45 \text { (86.5) } \\
\text { I } 3(5.8) \\
\text { S } 4(7.7) \\
\end{array}$ & $\begin{array}{l}\text { R } 10 \text { (62.5) } \\
\text { S } 6(37.5)\end{array}$ \\
\hline Gentamicin & 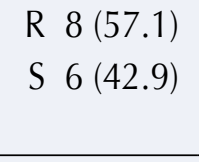 & $\begin{array}{c}\text { R } 33 \text { (66) } \\
\text { I } 1 \text { (2) } \\
\text { S } 16(32) \\
\end{array}$ & $\begin{array}{r}\text { R } 38 \text { (64.4) } \\
\text { I } 4(6.8) \\
\text { S } 17(28.8) \\
\end{array}$ & $\begin{array}{r}\text { R } 38(50) \\
\text { I } 2(7.1) \\
\text { S } 12(42.9) \\
\end{array}$ & $\begin{array}{r}\text { R } 9(56.2) \\
\text { I } 1(6.3) \\
\text { S } 6(37.5) \\
\end{array}$ \\
\hline Ciprofloxacin & $\begin{array}{l}\text { R } 7 \text { (50) } \\
\text { S } 7 \text { (50) }\end{array}$ & $\begin{array}{l}\text { R } 34(68) \\
\text { S } 16(32)\end{array}$ & $\begin{array}{c}\text { R } 38(64.4) \\
\text { I } 1(1.7) \\
\text { S } 20(33.9) \\
\end{array}$ & $\begin{array}{l}\text { R } 40(77) \\
\text { S } 12(23)\end{array}$ & $\begin{array}{lll}\text { R } & 10 & (62.5) \\
\text { I } & 1 & (6.2) \\
\text { S } & 5 & (31.3) \\
\end{array}$ \\
\hline Imipenem & $\begin{array}{c}\text { R } 3 \text { (21.4) } \\
\text { I } 1(7.1) \\
\text { S } 10(71.5) \\
\end{array}$ & $\begin{array}{l}\text { R } 29(58) \\
\text { I } 5(10) \\
\text { S } 16(32)\end{array}$ & $\begin{array}{l}\text { R } 35 \text { (59.3) } \\
\text { I } 1(1.7) \\
\text { S } 23(39)\end{array}$ & $\begin{array}{r}\text { R } 40(77) \\
\text { I } 2(3.8) \\
\text { S } 10(19.2) \\
\end{array}$ & $\begin{array}{lll}\text { R } 10 & (62.5) \\
\text { S } & 6 & (37.5)\end{array}$ \\
\hline Meropenem & $\begin{array}{r}\text { R } 3(21.4) \\
\text { I } 2(14.3) \\
\text { S } 9(64.3)\end{array}$ & $\begin{array}{r}\text { R } 28(56) \\
\text { I } 5(10) \\
\text { S } 17(34)\end{array}$ & $\begin{array}{c}\text { R } 35(59.3) \\
\text { I } 3 \quad(5.1) \\
\text { S } 21(35.6) \\
\end{array}$ & $\begin{array}{c}\mathrm{R} 40(77) \\
\mathrm{I} \quad 2(3.8) \\
\mathrm{S} 10(19.2)\end{array}$ & $\begin{array}{l}\text { R } 10(62.5) \\
S \quad 6(37.5)\end{array}$ \\
\hline Colistin & S 14 (100) & S $50(100)$ & S 59 (100) & $\begin{array}{r}\text { R } 1(1.9) \\
\text { S } 51(98.1) \\
\end{array}$ & S 16 (100) \\
\hline Tigecycline & NA & NA & $\begin{array}{cc}\text { R } 2 & (3.4) \\
\text { I } 1 & (1.7) \\
\text { S } 56 & (94.9)\end{array}$ & $\begin{array}{c}\text { R } 1(1.9) \\
\text { I } 1(1.9) \\
\text { S } 50(96.2)\end{array}$ & $\begin{array}{l}\text { R } 2(12.4) \\
\text { I } 3(18.8) \\
\text { S } 11(68.8)\end{array}$ \\
\hline
\end{tabular}

R: resistant, I: intermediate resistance, S: sensitive, NA: not applicable since it was not tested.

Table III. Distribution of MDR and non-MDR

A. baumannii isolates by years

\begin{tabular}{lrrr} 
Year & $\begin{array}{r}\text { MDR } \\
\mathrm{n}=132(\%)\end{array}$ & $\begin{array}{r}\text { Non-MDR } \\
\mathrm{n}=59(\%)\end{array}$ & $\begin{array}{r}\text { Total } \\
\mathrm{n}(\%)\end{array}$ \\
\hline $\mathbf{2 0 0 6}$ & $8(57)$ & $6(43)$ & $14(100)$ \\
\hline $\mathbf{2 0 0 7}$ & $32(64)$ & $18(36)$ & $50(100)$ \\
\hline $\mathbf{2 0 0 8}$ & $40(67.7)$ & $19(32.3)$ & $59(100)$ \\
\hline $\mathbf{2 0 0 9}$ & $42(80.7)$ & $10(19.3)$ & $52(100)$ \\
\hline $\mathbf{2 0 1 0}$ & $10(62.5)$ & $6(37.5)$ & $16(100)$ \\
\hline Total & $\mathbf{1 3 2 ( 6 9 )}$ & $\mathbf{5 9 ( 3 1 )}$ & $\mathbf{1 9 1 ( 1 0 0 )}$ \\
\hline
\end{tabular}

the antibiotic susceptibility of $A$. baumannii causing bacteraemia in our hospital. Over around four years, 191 case of bacteraemia due to $A$. baumannii have been reported at our hospital. Bacteraemia caused by $A$. baumannii was reported in adult group $(82.2 \%)$ as well as in paediatric age group (15.8\%). Invasive Acinetobacter infections is mostly health-care associated which may manifest as bacteraemia in adults, and usually as bacteraemia and/or meningitis in paediatric age group. ${ }^{5,9,20,21}$ Our results demonstrated that the majority of cases of bacteraemia were observed in critically ill patients, being the highest with patients in the surgical ICU. The oncology unit and $\mathrm{CCU}$ reported the next most common occurrence of $A$. 


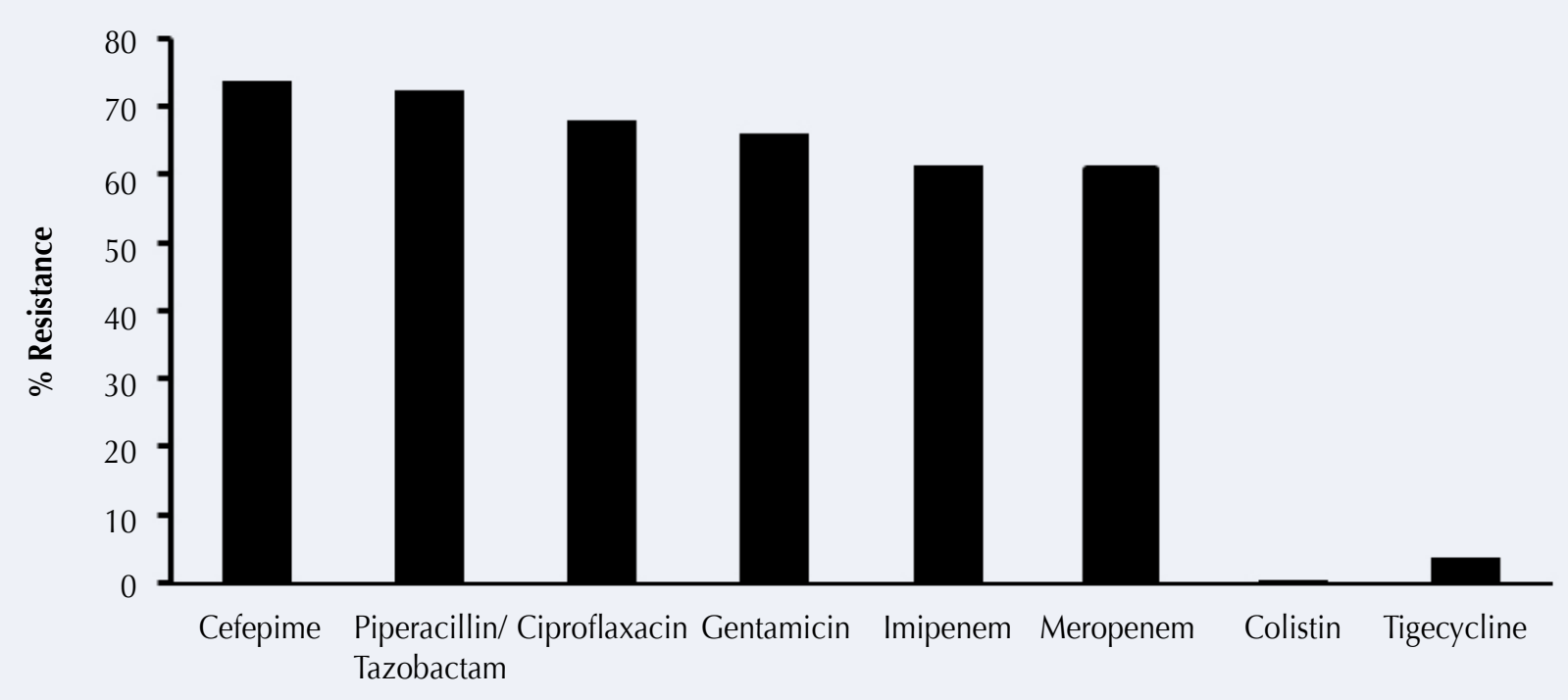

\section{Antimicrobial agents}

Figure 1. Resistance patterns of $A$. baumannii to antimicrobial agents

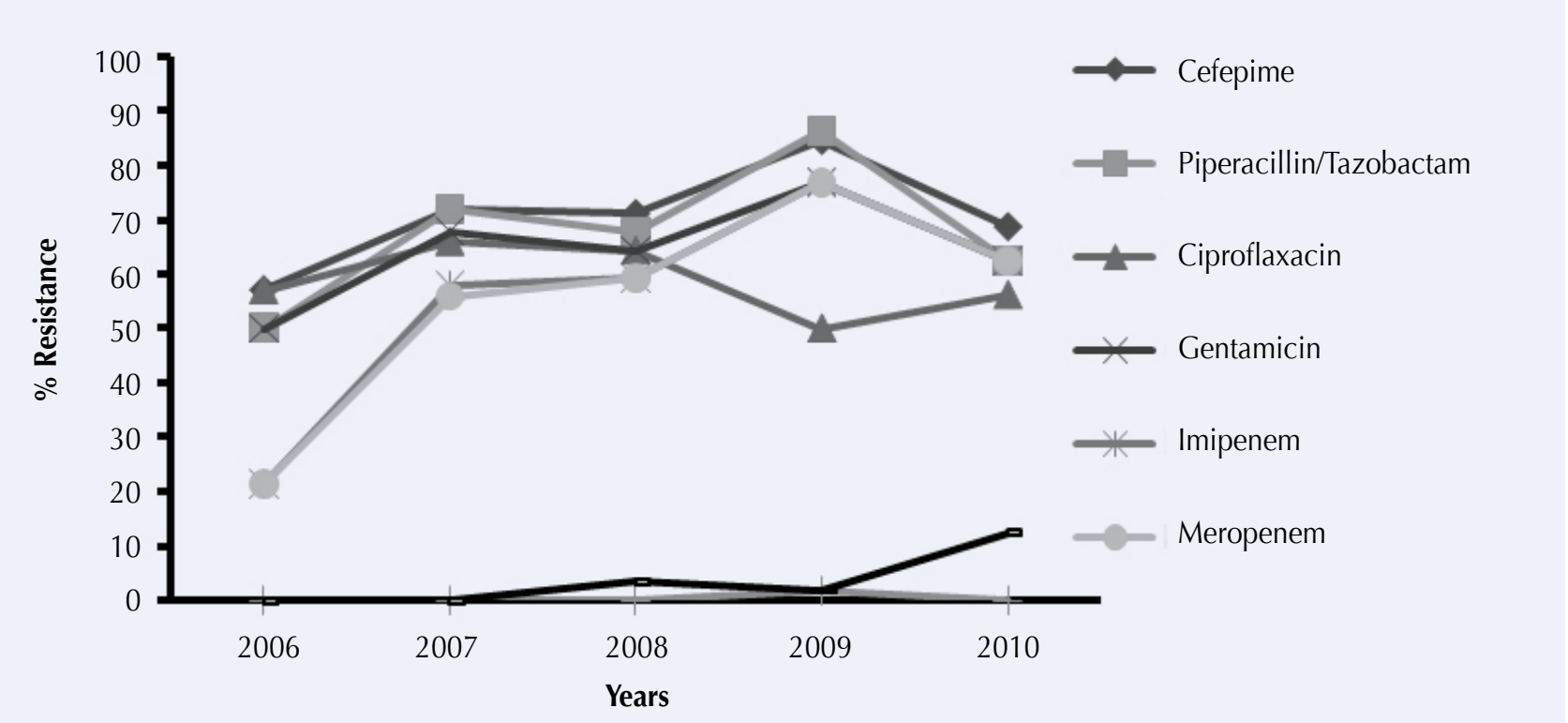

Figure 2. Annual resistance pattern of $A$. baumannii to antimicrobial agents

baumannii bacteraemia. This observation is consistent with other studies where they demonstrated that the highest occurrences of Acinetobacter spp. infections were from surgical ICU. $8,9,22$

In this study, it has been observed that $A$. baumannii was isolated either as a pure culture of bacteria, or as mixed culture with concomitant growth of other type of bacteria isolated from blood samples of our patients. Bacteria causing the co-infection were of both gramnegative and gram-positive. This is consistent with other reports were co-infection with $A B$ has been reported in different sites of the body. ${ }^{9,22}$ This type of co-infection may add to the burden of antibiotic resistance of these bacteria. However, Dent et al., demonstrated that this co-infection did not increase the mortality rate. ${ }^{9}$

Acinetobacter have the ability to develop resistance rapidly, and this has been observed particularly for carbapenems in different parts of the world. ${ }^{23-25} \mathrm{~A}$ number of antimicrobial resistance mechanisms 
have been implicated. These include chromosomally encoded B-lactamase (particularly AmpC cephalosporinase), OXA B-lactamases, metalloB-lactamases, efflux pump and integrons., ${ }^{1,12}$ A major problem of MDR $A$. baumannii is the extended resistance to aminoglycoside as well as quinolone groups. In this study, high resistance levels of $A$. baumannii to the commonly used antibiotics has been observed. The highest was for cefepime, followed by piperacillin / tazobactam, ciprofloxacin, gentamicin, imipenem, and meropenem respectively. In a recent study, authors reported that the increase in consumption of carbapenem was significantly related to the development of resistance in A. baumannii to imipenem and meropenem. ${ }^{26}$ They also reported that high consumption of carbapenem has been associated with resistance to piperacillin/tazobactam, ceftazidime, cefepime, amikacin, and levofloxacin. ${ }^{26}$ This high level of resistance to antibiotics observed in our study was not reported before in Saudi Arabia. Babay et al. in 2003 reported $100 \%$ susceptibility of A. calcoaceticus-baumannii complex to imipenem and around $69 \%$ for gentamicin, ceftazidime and ciprofloxacin. ${ }^{15}$ A later study conducted by AlTawfiq and Mohanhas, who evaluated the resistance pattern of $A$. calcoaceticus-baumannii complex at one hospital in Saudi Arabian, reported the maximum rates of resistance of $74.4 \%$ for ceftriaxone followed by $38.3 \%$ for imipenem. ${ }^{16}$ Moreover, they found an association of increasing resistance of this bacteria with the use of these antibiotics over the years. Higher and more alarming resistance rates came from a study conducted in Jordan. ${ }^{27}$ They reported that around $70 \%$ of isolates of $A$. baumannii were resistant to imipenem and meropenem.

An increasing prevalence of infections caused by MDR $A$. baumannii isolates has been reported in many countries. ${ }^{3,9,12,13,28,29}$ The emergence of MDR $A$. baumannii usually complicates treatment and leave clinicians with limited options. In this study, we found an increasing proportion of MDR A. baumannii isolates with years starting from 2006 till the end of 2009. A lower proportion of MDR was reported for the year 2010 after the alarming increase in the proportion of MDR in the year 2009. This might be explained by the fact that those reported for the year 2010 represent a period of 4 months only of this year.
Our study was designed to collect data for four years and since the hospital started functioning April 2006, we collected data till April 2010. This could be one of the limitations in which the data in the year 2010 were not completed for the whole year. A comparative rate of MDR A. baumannii has been reported by Dent et al. ${ }^{9}$ Using the same definition for MDR, they reported that $72 \%$ of $A$. baumannii isolates, recovered from 164 patients were MDR. ${ }^{9}$ In Germany, a similar rise in the numbers of MDR A. baumannii has been observed in four different hospitals. ${ }^{19}$ However, percentages reported in their study were much lower than the ones reported at our hospital. The cause of high rates of MDR strains observed in our study is not exactly known since the study design does not enable us to have an answer for this observation. However, one study has demonstrated that high resistance rates to imipenem and carbapemen were significantly correlated with resistance rates to ß-lactams, aminoglycosides and fluoroquinolones. ${ }^{26}$ Our study has several limitations which include: the percentages reported for resistance of $A$. baumannii to colistin and tigecycline could be affected by the fact that we did not start using or testing these antibiotics before the year 2008. The design of this study was a retrospective one and the limitations with this kind of study are well documented. ${ }^{30}$

In conclusion, $A$. baumannii is developing resistance to antibiotics extremely rapidly. This study highlighted the high and alarming resistance pattern of $A$. baumannii causing bacteraemia to the currently used antibiotics, particularly to carbapenems. Results from our study suggest encouraging clinicians for strict adherence to infection control policies and procedures (especially those of hand hygiene) with particular vigilance needed by the surgical team in surgical ICU to prevent outbreaks. In addition, enhancement of the antimicrobial stewardship program in the hospital which started in 2008 (The committee include multidisciplinary team of clinicians from infectious disease, infection control, microbiology specialties and clinical pharmacy). A more restrictive use of recommended antibiotics according to susceptibility testing and local guidelines is also required in order to slow the development of bacterial resistance. National multicenter studies are also encouraged to show the size of the problem of $A$. baumannii resistance to antimicrobials. The use of molecular 
methods to determine the resistance mechanisms involved in specific antimicrobial susceptibility profile is becoming a necessity. These will be of great help in establishing the local antibiotic and infection control policies in hospitals.

\section{Acknowledgments}

The authors would like to thank the staff members of Microbiology laboratory and Infection Control Department at KFMC Hospital for their assistance in data entry.

\section{References}

1. Peleg AY, Seifert H, Paterson DL. Acinetobacter baumannii: Emergence of a successful pathogen. Clin Microbiol Rev 2008; 21: 538-582. http://dx.doi.org/10.1128/CMR.00058-07

2. Hsueh PR, Teng LJ, Chen CY, et al. Pandrug-Resistant Acinetobacter baumannii Causing Nosocomial Infections in a University Hospital, Taiwan. Emerging Infectious Diseases 2002; 8: 827-832. http://dx.doi.org/10.3201/eid0808.020014

3. Faisal SA, Imran A, Fatima M, Rehan AS, Zaidi AKM. Panresistant Acinetobacter infection in neonates in Karachi, Pakistan. J Infect Dev Ctries 2010; 4(1): 30-37.

4. Panhotra BR, Saxena AK, Al-Mulhim AS. Contamination of patients' files in intensive care units: An indication of strict hand washing after entering case notes. American Journal of Infection Control 2005; 33: 398-401. http://dx.doi. org/10.1016/j.ajic.2004.12.009

5. Maragakis LL, Perl TM. Acinetobacter baumannii: Epidemiology, Antimicrobial Resistance, and Treatment Options. Clin Infect Dis 2008; 46: 1254-1263. http://dx.doi. org/10.1086/529198

6. Vallés J, León C, Alvarez-Lerma F. Nosocomial bacteremia in critically ill patients: a multicenter study evaluating epidemiology and prognosis. The Spanish Collaborative Group for Infections in Intensive Care Unit of SEMIUC. Clin Infect Dis 1997; 24: 387-395. http://dx.doi.org/10.1093/ clinids/24.3.387

7. Pittet D, Tarara D, Wenzel RP. Nosocomial bloodstream infections in critically ill patients: excess length of stay, extra costs, and attributable mortality. JAMA 1994; 271: 1598-1601. http://dx.doi.org/10.1001/jama.1994.03510440058033

8. A'Garmendia JLuis G, Ortiz-Leyba C, Garnacho-Montero J, et al. Risk Factors for Acinetobacter baumannii Nosocomial Bacteremia in Critically III Patients: A Cohort Study. Clin Infect Dis 2001; 33: 939-946. http://dx.doi.org/10.1086/322584

9. Dent LL, Marshall DR, Pratap S, Hulette RB. Multidrug resistant Acinetobacter baumannii: a descriptive study in a city hospital. BMC Infect Dis 2010; 7: 196. http://dx.doi.org/10.1186/14712334-10-196

10. Zavascki AP, Carvalhaes CG, Picão RC, Gales AC. Multidrugresistant Pseudomonas aeruginosa and Acinetobacter baumannii: resistance mechanisms and implications for therapy. Expert Rev Anti Infect Ther 2010; 8: 71-93. http:// dx.doi.org/10.1586/eri.09.108

11. Fishbain J, Peleg AY. Treatment of Acinetobacter infections. Clin Infect Dis 2010; 51: 79-84. http://dx.doi.org/10.1086/653120

12. Gordon NC, Wareham DW. Multidrug-resistant Acinetobacter baumannii: mechanisms of virulence and resistance. International Journal of Antimicrobial Agents 2010; 35: 219226. http://dx.doi.org/10.1016/j.ijantimicag.2009.10.024
13. Falagasa ME, Rafailidis $\mathrm{Pl}$, loannidoua $\mathrm{E}$, et al. Colistin therapy for microbiologically documented multidrug-resistant Gram-negative bacterial infections: a retrospective cohort study of 258 patients. International Journal of Antimicrobial Agents 2010; 35: 194-199. http://dx.doi.org/10.1016/j. ijantimicag.2009.10.005

14. Poirel L, Nordmann P. Carbapenem resistance in Acinetobacter baumannii: mechanisms and epidemiology. Clin Microbiol Infect 2006; 12: 826-836. http://dx.doi.org/10.1111/j.14690691.2006.01456.x

15. Babay HA, Kambal AM, Al-Anazy AR, Saidu AB, Aziz S. Acinetobacter blood stream infection in a teaching hospital - Riyadh, Saudi Arabia. Kuwait Medical Journal 2003; 35: 196201.

16. Al-Tawfiq JA, Mohandhas TX. Prevalence of antimicrobial resistance in Acinetobacter calcoaceticus-baumannii complex in a Saudi Arabian hospital. Infection control and hospital epidemiology 2007; 28: 870-872. http://dx.doi. org/10.1086/518842

17. National Committee for Clinical and Laboratory Standards (NCCLS). Methods for Dilution Antimicrobial Susceptibility Tests for Bacteria that Grow Aerobically. Approved Standard. 6th ed. Wayne, PA: NCCLS; 2003: M7-A6.

18. Garnacho-Montero J, Amaya-Villar R. Multiresistant Acinetobacter baumannii infections: epidemiology and management. Curr Opin Infect Dis 2010; 23: 332-339. http:// dx.doi.org/10.1097/QCO.0b013e32833ae38b

19. Wadl M, Heckenbach K, Noll I, et al. Increasing occurrence of multidrug-resistance in Acinetobacter baumannii isolates from four German University Hospitals, 2002-2006. Infection 2010; 38: 47-51. http://dx.doi.org/10.1007/s15010-009-9225-x

20. Egbe CA, Ndiokwere C, Omoregie R. Microbiology of lower respiratory tract infections in Benin city, Nigeria. Malays I Med Sci 2011; 18: 27-31.

21. Hu J, Robinson JL. Systematic review of invasive Acinetobacter infections in children. Can J Infect Dis Med Microbiol 2010; 21: 83-88.

22. Chua MM, Alejandria MM. The epidemiology of Acinetobacter infections among critically ill adult patients admitted at the University of the Philippines - Philippine General Hospital. Philippine Journal of Microbiology and Infectious Diseases 2008; 37: 38-53.

23. Bergogne-Berezin E, Towner KJ. Acinetobacter spp. as nosocomial pathogens: microbiological, clinical, and epidemiological features. Clin Microbiol Rev 1996; 9: 148165.

24. Martins AF, Kuchenbecker R, Sukiennik T, et al. Carbapenemresistant Acinetobacter baumannii producing the OXA-23 enzyme: dissemination in Southern Brazil. Infection 2009; 37: 474-476. http://dx.doi.org/10.1007/s15010-009-9003-9

25. Kulah C, Mooij MJ, Comert F, et al. Characterisation of carbapenem-resistant Acinetobacter baumannii outbreak strains producing OXA-58 in Turkey. Int / Antimicrob Agent 2010; 36: 114-118. http://dx.doi.org/10.1016/j. ijantimicag.2010.03.017

26. Cao J, Song W, Gu B, et al. Correlation between carbapenem consumption and antimicrobial resistance rates of Acinetobacter baumannii in a University-Affiliated Hospital in China Hong Zhou. J Clin Pharmacol 2013; 53(1): 96-102. http://dx.doi.org/10.1177/0091270011435988

27. Dhabaan GN, Hamimah H, Shorman MA. Emergence of extensive drug-resistant Acinetobacter baumannii in North of Jordan. African Journal of Microbiology Research 2011; 5: 1070-1075. 
28. Kempf M, Rolain JM. Emergence of resistance to carbapenems in Acinetobacter baumannii in Europe: clinical impact and therapeutic options. Int / Antimicrob Agents 2012; 39: 105114. http://dx.doi.org/10.1016/j.ijantimicag.2011.10.004

29. Routsi C, Pratikaki M, Platsouka E, et al. Carbapenem-resistant versus carbapenem-susceptible Acinetobacter baumannii bacteremia in a Greek intensive care unit: risk factors, clinical features and outcomes. Infection 2010; 38: 173-180. http:// dx.doi.org/10.1007/s15010-010-0008-1

30. Hess DR. Retrospective studies and chart reviews. Respir Care 2004; 49(10): 1171-1174. 